
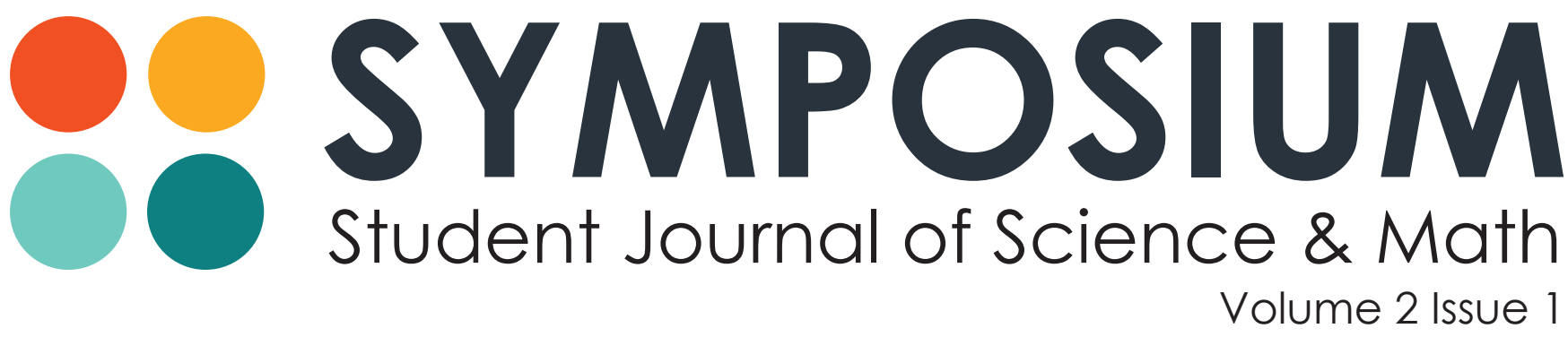


\section{VARIATION IN MICROBIOME COMPOSITION AND STABILITY FOR A VANCOMYCIN TREATED PRIMARY SCLEROSING CHOLANGITIS PATIENT WITH ULCERATIVE COLITIS COMPARED WITH CONTROLS}

A RESEARCH ARTICLE

by Alanna Dubrovsky

\section{Abstract}

The bacterial composition of the human intestines contributes to much more than just digestion. In the inflammatory autoimmune conditions primary sclerosing cholangitis (PSC) and ulcerative colitis (UC), the microbiome may be, in some cases, a factor. To gain a better understanding of the composition and stability of the microbiome in a patient treated with vancomycin for PSC, terminal restriction fragment (TRF) analysis was performed on $\mathrm{I}_{3}$ controls and I patient, and I6s rRNA microbiome composition analyses were performed on I patient and 3 controls. Results showed similar levels of stability with surprising differences in composition.

\section{Introduction}

Accompanying the recent increase in autoimmune and gastrointestinal diseases in developed nations is a spike in interest in the microbial composition of the intestines. With emerging evidence implying the microbiome's role in diseases beyond the reach of the digestive system, such as autism, many researchers from varying areas of study are finding further implications of a fluctuating and unusual microbiome (4). Two of the many diseases linked to microbiome abnormalities are primary sclerosing cholangitis (PSC) and ulcerative colitis (UC) (5). Ulcerative Colitis is an autoimmune disease characterized by inflammation of the large intestine. Primary sclerosing cholangitis is another autoimmune disease in which immune cells attack the bile ducts of the liver, causing liver disease and, in many cases, liver 
failure. Researchers have hypothesized in the leaky gut theory that these two autoimmune diseases are linked together through microbial effects in the gut (6). In association with this theory, the antibiotic vancomycin became an effective treatment option for PSC when initiated in childhood. However, treatment is not effective when treatment begins in adult patients. The basis for this peculiar specificity is still a burning question for doctors and researchers.

Vancomycin treatment was also effective for both UC and PSC in some patients with both diagnoses. This leads to the question: what distinguishes patients whose UC responds to vanomycin treatment from those that do not? A piece to this puzzle may be the variation in microbial flora of these two types of patients. This study was conducted to examine the microbiome composition and stability in a patient with PSC and UC by comparing terminal restriction fragment (TRF) pattern peaks to $\mathrm{I} 3$ normal control patients, and microbiome sequencing to 3 of the normal control patients.

\section{Materials \& Methods}

In this study, stability analysis of TRF patterns was performed for one UC/PSC patient and I3 control patients. Bacterial microbiome composition analysis was done on the same UC/PSC patient treated with vancomycin for two microbiome samples of different days and 3 control patients.

\section{Fecal Sample Collection:}

Subjects were provided with sterile, I.5 mL tubes for sample collection. Fecal samples were collected on plastic wrap and transferred using a sterile toothpick into the sterile tube. Tubes were filled approximately $\mathrm{I} / 3$ of the way full and stored at $-20^{\circ} \mathrm{C}$ until DNA extraction. This process was repeated for I 4 consecutive days.

\section{DNA Extraction:}

A MoBio Powersoil ${ }^{\otimes}$ DNA kit was used to extract DNA from samples following manufacturer's instructions. To measure the success of the DNA extraction, the sample concentrations were quantified using a spectrophotometer.

\section{PCR Amplification and Cleanup for TRF:}

Triplicate PCR was performed using I6s rRNA sequences homologous to eubacterial regions. For each reaction, $24 \mu \mathrm{L}$ of master mix was combined with $3 \mu \mathrm{L}$ of extracted DNA. The master mix contained 5 $\mu \mathrm{L}$ of Io $\mu \mathrm{L} 5 \mathrm{X}$ GoTaq buffer; I. $5 \mu \mathrm{L}$ of Io mM dNTPs; I $\mu \mathrm{L}$ of $20 \mu \mathrm{g} / \mathrm{mL}$ BSA; $3.5 \mu \mathrm{L}$ of $25 \mathrm{mM} \mathrm{MgCl} 2$; $0.5 \mu \mathrm{L}$ of IoM labeled $8 \mathrm{dF}$; 0.5 $\mu \mathrm{L}$ of Io $\mu \mathrm{M} \mathrm{K} \mathrm{K}_{2} \mathrm{R}$; 1 II.85 $(\mathrm{L}$ of purified PCR water; and 0.15 ( $\mathrm{L}$ of 5 $\mathrm{U} / \int \mathrm{L}$ of AmpliTaq Gold. The forward primer used was 8dF (AGAGTTTGTTCMTGGCTCAG), and the reverse primer was $\mathrm{K}_{2} \mathrm{R}$ (GTATTACCGCGGCTGCTGG). The parameters for the PCR cycle were as follows: the initiating step was set at $94^{\circ} \mathrm{C}$ for Io minutes; the denaturing step at $94^{\circ} \mathrm{C}$ for I minute; the annealing step at $46.5^{\circ} \mathrm{C}$ for I minute; the elongation step at $72^{\circ} \mathrm{C}$ for 2 minutes; the final elongation at $72{ }^{\circ} \mathrm{C}$ for Io minutes; and the final hold at $4^{\circ} \mathrm{C}$. A sample of $E$. coli DNA was used as a control for all reactions. The success of each reaction was confirmed using gel electrophoresis on I\% agarose gels containing ethidium bromide as a DNA fluorescent tag. Triplicate amplified samples were combined before PCR cleanup. MoBio PCR Ultra-Clean kit was used for PCR cleanup. 


\section{Enzyme Digest and Ethanol Precipitation:}

For the enzyme digest, $0.4 \mu \mathrm{L}$ of Hae III enzyme and $4 \mu \mathrm{L}$ of buffer were combined with enough DNA sample and purified water to bring the sample to a volume of $40 \mu \mathrm{L}$. The amount of DNA sample varied based on measured concentrations and previous success of digest on the samples, but was generally between 75-300 ng. An E. coli control sample was made using 5 ng of E. coli DNA. The samples were placed in the PCR machine for 4 hours at $37^{\circ} \mathrm{C}$, then 20 minutes at $80^{\circ} \mathrm{C}$, and $4^{\circ} \mathrm{C}$ until removal from the PCR machine for storage. For ethanol precipitation, Ioo $\mu \mathrm{L}$ of $95 \%$ ethanol, $2 \mu \mathrm{L}$ of ${ }_{3} \mathrm{M}$ Sodium Acetate ( $\mathrm{pH}=4.6$ ), and I $\mu \mathrm{L}$ of glycogen were added to each digested sample. The samples were inverted several times, cooled for 30 minutes, and placed in a centrifuge for 15 minutes at 5300 RPM. Samples were immediately inverted onto a paper towel, combined with 100 $\mu \mathrm{L}$ of $70 \%$ ethanol, and centrifuged for 5 minutes at $5300 \mathrm{rpm}$. Samples were once again immediately inverted and centrifuged in an inverted position for I minute at $700 \mathrm{rpm}$.

\section{TRF Pattern Generation:}

Digested DNA samples were resuspended in $20 \mu \mathrm{L}$ of formamide and $0.25 \mu \mathrm{L}$ of CEQ 600 base pair standard. TRF patterns were generated using the Beckman Coulter CEQ 8000X DNA analysis system. Bray Curtis similarity analysis was performed on the data, and a multidimensional scaling and cluster dendogram were created from the similarity data using Primer 5 software.

\section{DNA Sequencing:}

Extracted DNA samples from day io for two controls and day 8 for one control, along with samples from day II and 13 from the treated patient, were sent to MR DNA labs in Shallowater, Texas for DNA analysis. The chosen samples showed diversity in species present as determined by TRF patterns. Primers designated " 515 " and "806" (referring to I6s rRNA base numbers) were used with a Personal Genome Machine (PGM) analysis for DNA classification, and data were converted into percentages of total sequences.

\section{Results}

\section{TRF pattern similarity}

TRF patterns were generated for each of the samples collected and analyzed for similarities within a subject and between subjects. TRF pattern generation allows a direct comparison of the varying microbes present and the relative abundance of each. The characteristic restriction site results in a nucleotide fragment representative of an individual microbial species. The TRF patterns for the treated patient showed less variation than those of the control subjects, with one or two main peaks for each day (Fig I). The prominent TRF peaks observed in control subjects were conserved across days in each subject. Control subject TRF patterns were generally similar to one another, while patient samples differed remarkably from the control cluster (Fig 2). However, TRF patterns for the treated patient's samples were generally very similar to one another over the I4-day sampling period, with an average similarity of $62.6 \%$ (Fig 3 ).

TRF peaks were consistent over the I4 days of sampling for all the samples in the control subjects 
and the treated patient. Food logs were recorded to account for any instability observed, but it was not needed after analysis.

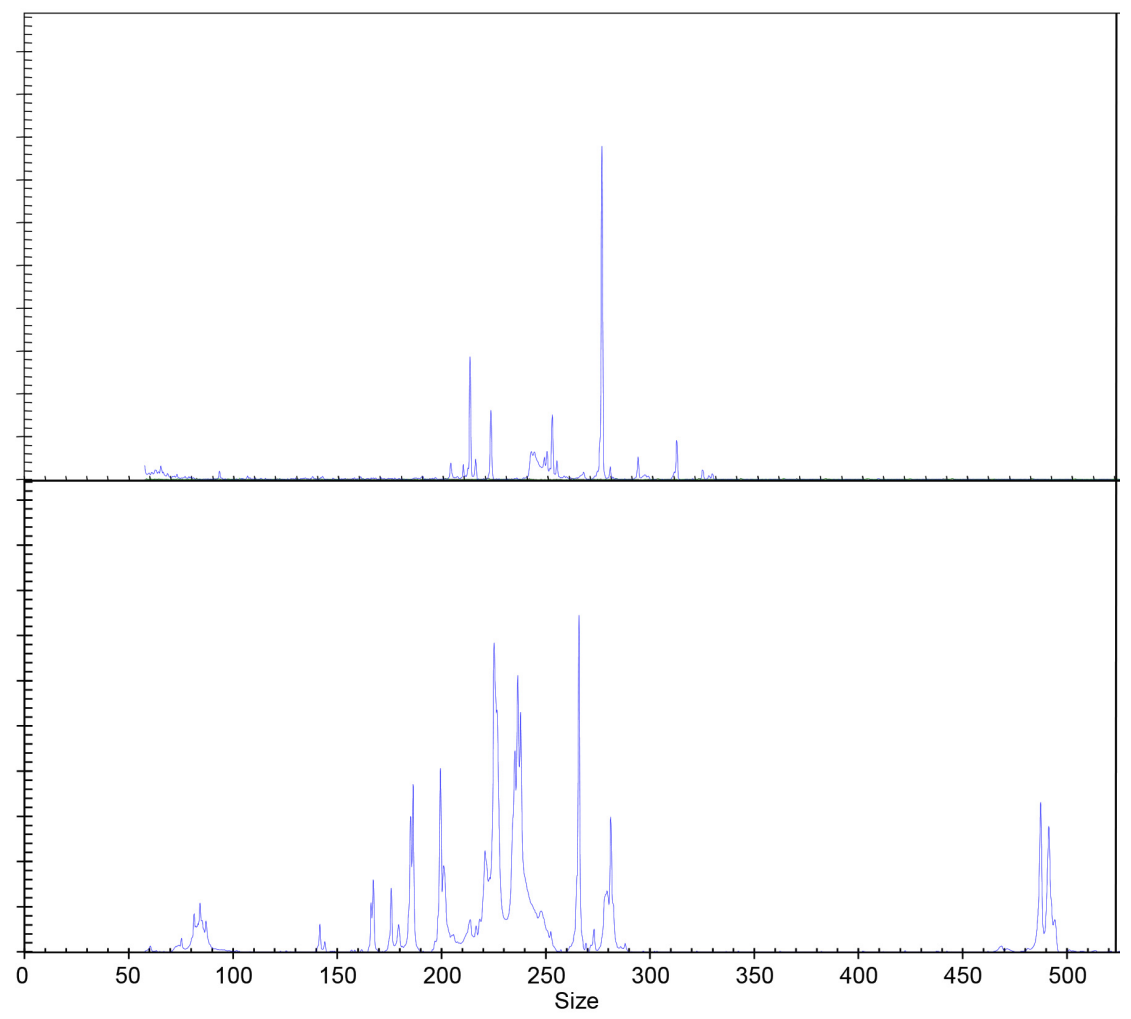

Figure I. TRF pattern (raw data) comparison between patient (top) and control (bottom).

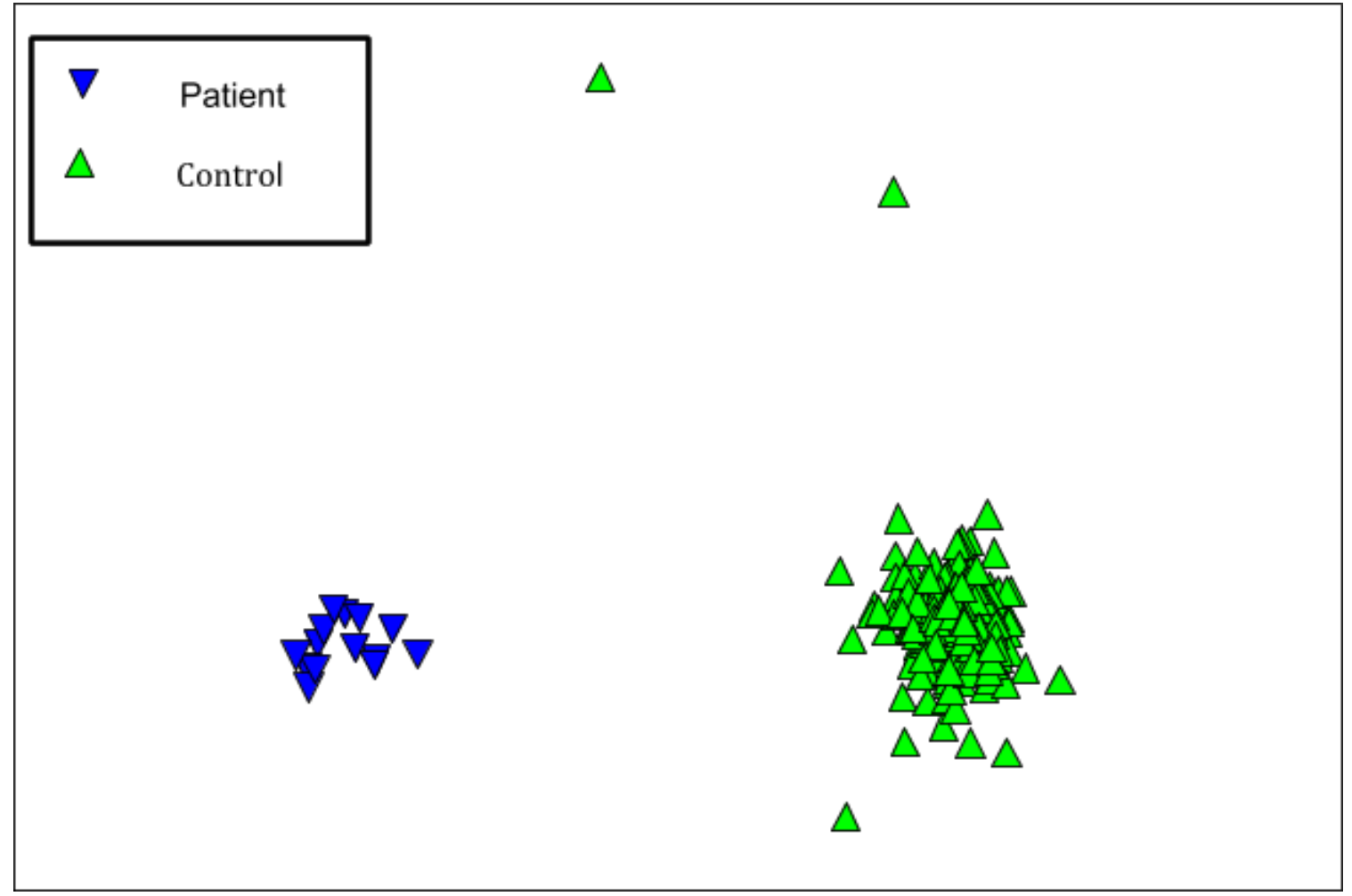

Figure 2. Multidimensional scaling analysis on TRF pattern similarity using Bray Curtis comparing I4 days of samples for the patient and the I4 days of samples for each control. 


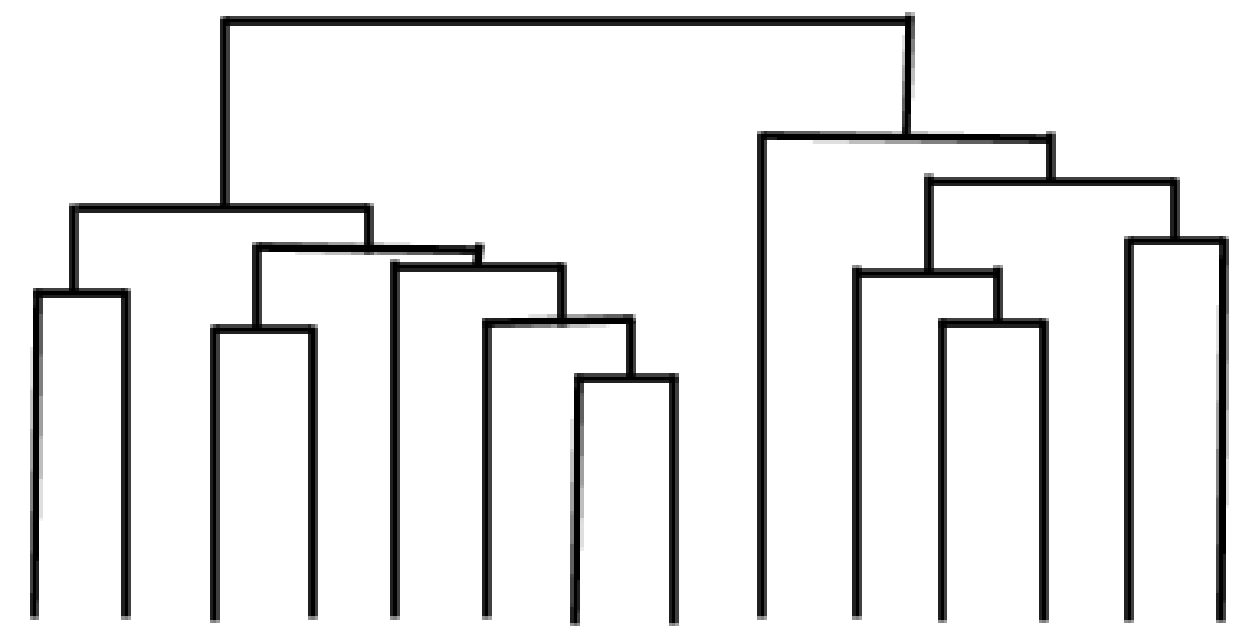

Figure 3. Dendogram of TRF peak similarity between the I4 days of the patient's samples.

\section{Microbial composition variation}

To gain a more comprehensive understanding of the variations between control subjects and the treated patient, microbial sequencing was performed. There was a noteworthy shift at day 8 for the patient, with similarity less than 50 percent between day 8 and day 9. Microbial composition of fecal samples varied dramatically between control subjects and the two samples from the treated patient. The primary difference is the dominance of Fusobacterium ulcerans seen in both samples from the treated patient, with $79 \%$ on day II and $52 \%$ on day I3, while all control subjects had considerably lower values, with a high of $0.0016 \%$. The other apparent difference is seen in the percentages of Blautia present, with an average of II\% for control subjects and 0.00I9\% for the treated patient (Table I). There were minimal differences between the control subjects, likely due to diet and lifestyle, with no noteworthy variation observed. Notably, the majority of species present in large percentages in controls, but absent in the patient samples, were gram-positive bacterial species.

\section{Discussion}

The causes for both UC and PSC are very poorly understood, and treatments for PSC are lacking. To better understand the communication between the two diseases, it is vital to understand why the use of the antibiotic vancomycin would treat symptoms and reverse liver damage caused by PSC. The consistency of the TRF patterns, indicating stability of microbial communities, shows that the natural microbiome is stable across a I4-day period, provided no unusual circumstances are present. There was limited additional stabilizing effect observed from the vancomycin treatment, based on the TRF 
results, with 57.9 average percent similarity for controls and 62.6 for the patient. The cause of the low similarity between day 8 and 9 is unknown, with no significant lifestyle changes between these days.

The causes for both UC and PSC are very poorly understood, and treatments for PSC are lacking. To better understand the communication between the two diseases, it is vital to understand why the use of the antibiotic vancomycin would treat symptoms and reverse liver damage caused by PSC. The consistency of the TRF patterns, indicating stability of microbial communities, shows that the natural microbiome is stable across a I4-day period, provided no unusual circumstances are present. There was limited additional stabilizing effect observed from the vancomycin treatment, based on the TRF results, with 57.9 average percent similarity for controls and 62.6 for the patient. The cause of the low similarity between day 8 and 9 is unknown, with no significant lifestyle changes between these days.

The microbial composition observed in the treated patient is characteristic of UC microbiome samples. The Fusobacteriaceae family is elevated in patients with inflammatory bowel disease (I). Although it is unknown whether this prevalence is a symptom of the disease or a pathogenic species leading to disease development, higher levels of antibodies against these bacterial species are recorded in UC studies (2).

The considerably lower values observed for certain bacteria in the vancomycin treated patient are likely due to vancomycin treatment. Vancomycin targets the D-Ala-D-Ala protein cell wall sequence, and therefore, gram-positive bacteria are particularly susceptible to vancomycin treatment (3). The decreased levels of the gram-positive bacteria Blautia in the treated patient offer support for this conclusion, as well as for the low levels of Subdoligranulum.

The next step for further understanding of vancomycin treatment and its effects on the microbiome will be to determine whether there is microbial variation in UC/PSC treated patients for whom both conditions are controlled with vancomycin, compared to treated patients for whom the vancomycin is only effective in controlling PSC, such as the patient studied. Since vancomycin is such an effective treatment for PSC, it is likely that one of the bacterial species eliminated with this treatment acts as a pathogenic species within the gut of pediatric patients. Once these species are removed from the intestinal environment, it is likely that other species will exploit the excess resources and expand their population. It is possible that the expanding species may vary between patients, leading to treatment of both UC and PSC with vancomycin in some patients. Further studies should involve observing the microbiomes of adult UC/PSC patients for differences between the composition of adult and pediatric patient microbiomes. In addition, it would be interesting to see if other gram-positive antibiotics, such as the new antibiotic teixobactin, would work in a similar way as vancomycin. This could be important if antibiotic resistance arises for vancomycin. 


\begin{tabular}{|c|c|c|c|c|c|c|c|c|}
\hline Family & Genus & Species & Gram & $\begin{array}{l}\text { Patient. } \\
\text { D11 }\end{array}$ & $\begin{array}{l}\text { Patient } \\
\text {.D13 }\end{array}$ & $\begin{array}{l}\text { Control } \\
1 . D 10\end{array}$ & $\begin{array}{l}\text { Control } \\
\text { 2.D10 }\end{array}$ & $\begin{array}{l}\text { Control } \\
\text { 3.D8 }\end{array}$ \\
\hline Synergistaceae & Cloacibacillus & Cloacibacillus spp. & - & 0.91 & 5.01 & 0.00 & 0.00 & 0.00 \\
\hline Eubacteriaceae & Enterobacter & $\begin{array}{l}\text { Enterobacter } \\
\text { hormaechei }\end{array}$ & - & 2.89 & 5.11 & 0.00 & 0.00 & 0.00 \\
\hline \multirow{4}{*}{ Clostridiaceae } & Subdoligranulum & Subdoligranulum spp. & + & 0.00 & 0.00 & 5.28 & 6.83 & 7.75 \\
\hline & \multirow{2}{*}{ Faecalibacterium } & $\begin{array}{l}\text { Faecalibacterium } \\
\text { prausnitzii }\end{array}$ & + & 0.00 & 0.00 & 7.76 & 8.11 & 0.00 \\
\hline & & Faecalibacterium spp. & + & 0.00 & 0.00 & 4.53 & 4.29 & 0.00 \\
\hline & Blautia & Blautia spp. & + & 0.00 & 0.00 & 13.87 & 7.61 & 11.40 \\
\hline \multirow[t]{2}{*}{ Lachnospiraceae } & Coprococcus & Coprococcus spp. & + & 0.00 & 0.00 & 3.36 & 1.08 & 2.77 \\
\hline & Ruminococcus & Ruminococcus spp. & + & 0.00 & 0.00 & 6.57 & 5.69 & 5.11 \\
\hline \multirow{2}{*}{ Enterobacteriaceae } & Pantoea & Pantoea agglomerans & - & 3.47 & 5.84 & 0.00 & 0.00 & 0.00 \\
\hline & Eubacterium & Eubacterium rectale & - & 0.00 & 0.00 & 4.49 & 4.01 & 4.38 \\
\hline Veillonellaceae & Veillonella & Veillonella dispar & - & 1.37 & 14.30 & 0.15 & 0.00 & 0.00 \\
\hline \multirow{4}{*}{ Bacteroidaceae } & \multirow{4}{*}{ Bacteroides } & Bacteroides vulgatus & + & 0.00 & 0.00 & 2.23 & 7.87 & 3.59 \\
\hline & & Bacteroides eggerthii & + & 0.00 & 0.00 & 10.20 & 1.43 & 0.00 \\
\hline & & Bacteroides uniformis & + & 0.00 & 0.00 & 3.19 & 5.36 & 8.42 \\
\hline & & Bacteroides stercoris & + & 0.00 & 0.00 & 0.02 & 10.26 & 2.18 \\
\hline \multirow[b]{2}{*}{ Fusobacteriaceae } & Cetobacterium & Cetobacterium somerae & - & 3.94 & 1.86 & 0.00 & 0.00 & 0.00 \\
\hline & Fusobacterium & $\begin{array}{l}\text { Fusobacterium } \\
\text { ulcerans }\end{array}$ & - & 79.15 & 51.67 & 0.00 & 0.00 & 0.00 \\
\hline Rikenellaceae & Alistipes & Alistipes putredinis & - & 0.00 & 0.00 & 0.39 & 2.72 & 2.33 \\
\hline
\end{tabular}

Table I. Percentages of important microbial distinctions between controls and treated subjects. 


\section{Cited Sources}

Courvalin, P. (2006). Vancomycin resistance in gram-positive cocci. Clinical Infectious Diseases, 42(Supplement I), S25-S34. doi:I0.I086/49I7II Fasano, A. (20I2). Leaky gut and autoimmune diseases. Clinical Reviews in Allergy \& Immunology, 42(I), 7I-78. doi:Io.IO07/sI20I6-OII-829I-X

Mulle, J., Sharp, W., \& Cubells, J. (2013). The gut microbiome: A new frontier in autism research. Current Psychiatry Reports, I5(2), $337-337$. doi:I0.1007/sII920-0I2-0337-0

Sasaki, M., \& Klapproth, J. (20I2). The role of bacteria in the pathogenesis of ulcerative colitis. Journal of Signal Transduction, 2012 , I-6. doi:IO.II55/2012/704953

Young, V. (n.d.). Ulcerative colitis human microbiome project (UCHMP). Retrieved from http://www.ncbi.nlm.nih.gov/projects/gap/cgibin/ study.cgi?study_id=phsooo262.vi.pI

Yukawa, T., Ohkusa, T., Shibuya, T., Tsukinaga, S., Mitobe, J., Takakura, K., ..., \& Tajiri, H. (2013). Nested culture method improves detection of fusobacterium from stool in patients with ulcerative colitis. Japanese Journal of Infectious Diseases, 66(2), I09-II4. Retrieved from http://www.ncbi.nlm.nih.gov/pubmed/23514906 